Check for updates

Cite this: RSC Adv., 2017, 7, 22946

Received 11th March 2017

Accepted 10th April 2017

DOI: 10.1039/c7ra02953e

rsc.li/rsc-advances

\section{Emulsion-derived hierarchically porous polystyrene solid foam for oil removal from aqueous environment $\uparrow$}

\begin{abstract}
Ning Zhang, Suting Zhong, Teng Chen, Yu Zhou and Wei Jiang (D)*
Herein, a high internal phase emulsion (HIPE) technique to fabricate hierarchically porous monolithic $\mathrm{Fe}_{3} \mathrm{O}_{4}$ /polystyrene composite material has been described. A novel co-stabilizer system of Span 20 and $\mathrm{Fe}_{3} \mathrm{O}_{4}$ solid particles was adopted to prepare the styrene-based emulsion. By adjusting the surfactant level, the concentration of $\mathrm{Fe}_{3} \mathrm{O}_{4}$ particles, and water content, a hierarchically interconnected porous material with a secondary pore structure of nano/micro size was constructed. In addition, the resulting composite polymer material exhibited excellent thermal stability, attributing to the good dispersion of $\mathrm{Fe}_{3} \mathrm{O}_{4}$ particles in the polymer matrix and strong bonding between solid particles and polymer chains. Moreover, the oil adsorption capacity of the solid foam was 16 times its own mass even after 10 cycles of oil/water separation. Importantly, the polystyrene solid foam exhibited hydrophobicity and oleophilicity without any further modification. Moreover, the incorporation of $\mathrm{Fe}_{3} \mathrm{O}_{4}$ particles allowed the composite material to be conveniently collected under an external magnetic field. All these features together make the polystyrene composite material an ideal adsorbent in the oil/water separation.
\end{abstract}

\section{Introduction}

Oil spill is a common and terrible phenomenon during the storage and transportation of oil resources, which has catastrophic effects on aquatic ecosystems and presents environmental hazard to humans..$^{\mathbf{1 - 4}}$ Moreover, among various methods, including oil adsorbents, ${ }^{5}$ filtration, ${ }^{6}$ bioremediation, skimmers, and in situ burning, for oil spill remediation, adsorption is considered to be ecologically friendly, economical, and one of the most efficient techniques. ${ }^{7}$ As a result, the development of cheap and reliable oil adsorbents is desirable and urgent both in the fields of fundamental research and practical applications. ${ }^{\mathbf{8}}$

High internal phase emulsions (HIPEs) is a template method to fabricate porous polymers, defined as PolyHIPEs, with controllable pore structures. ${ }^{9-11}$ The emulsion consists of an external continuous monomer phase and an internal droplet phase exceeding $74 \%$ of the total volume. ${ }^{\mathbf{1 2 - 1 4}}$ Because of their high porosity, large surface area, and interconnected pores, PolyHIPEs have great potential in the field of separation. ${ }^{\mathbf{1 5}}$

In general, HIPEs were stabilized via commercially available surfactants. ${ }^{16}$ According to Williams's report, Span 80 with a hydrophile-lipophile balance (HLB) of 4.3 was the most suitable surfactant to stabilize the styrene/divinylbenzene (St-DVB)

School of Chemical Engineering, Nanjing University of Science and Technology, Nanjing 210094, PR China. E-mail: superfine_jw@126.com

$\dagger$ Electronic supplementary information (ESI) available. See DOI: 10.1039/c7ra02953e
HIPE system. ${ }^{17}$ Moreover, Span 80 was also adopted in our previous research during the preparation of poly(St-DVB) foam, which exhibited excellent oil adsorption capacity in oil/water separation. ${ }^{18}$ To date, studies on the individual application of Span 20 as an emulsifier in the HIPE have not been reported because the HLB value of 8.6 is relatively high and the surfactant adsorbed at the interface is poor at preventing Ostwald ripening. However, studies on mixed surfactant systems, including Span 20, hexadecyltrimethylammonium bromide (CATB), and dodecylbenzenesulfonic acid sodium salt (DDBSS), as a co-stabilizer of the HIPEs can be found in the literature. ${ }^{17,19}$

Some solid particles due to their high energy of attachment ${ }^{20}$ can be irreversibly adsorbed at the interface of the two immiscible liquids, forming Pickering HIPEs. ${ }^{21-23}$ In addition, certain types of inorganic particles embedded into the polymeric matrix impart the resulting PolyHIPEs material with some additional properties ${ }^{24,25}$ such as mechanical, optical, thermal, magnetic or chemical properties. The ability of particles to adsorb at the interface between the two phases is primarily dependent on the wettability of the particles. ${ }^{25}$ Oleic acid is a common and effective agent to functionalize the particles with proper wettability. For example, zirconium dioxide was first modified with oleic acid and then used as a stabilizer to form Pickering HIPEs templates of St and DVB. ${ }^{22}$ Titania, ${ }^{26,27}$ silica, ${ }^{20,28}$ and $\mathrm{Fe}_{3} \mathrm{O}_{4}$ particles ${ }^{29,30}$ were all modified with oleic acid first and then used to stabilize the w/o emulsion to prepare the HIPEs. However, the modification process was tedious. ${ }^{31,32}$ In addition, it often generated macroporous polymers with typical closed-cell 
structures. Therefore, application of these closed-cell polymers in the field of oil/water separation was restrained.

To the best of our knowledge, the use of amphiphilic surfactant Span 20 together with solid particles to stabilize the water-in-oil HIPEs has not been reported to date. In this study, a novel system of two components, Span 20 and $\mathrm{Fe}_{3} \mathrm{O}_{4}$ particles, was used to fabricate the HIPEs template. On the one hand, the existence of amphiphilic surfactant Span 20 decreased the interfacial tension and improved the dispersion of the $\mathrm{Fe}_{3} \mathrm{O}_{4}$ particles in the emulsion system. On the other hand, $\mathrm{Fe}_{3} \mathrm{O}_{4}$ particles adsorbed at the interface and formed a rigid film, retarding the creaming of the oil drops or sedimentation of the water drops. ${ }^{33}$ The synergism of the two components played an important role in the effective emulsification and stabilization of the HIPEs. Importantly, the obtained PolyHIPEs material had interconnected and hierarchically porous structures. Moreover, it has been reported that emulsions stabilized by carbonaceous microsphere particles and surfactant Span 80 simultaneously produced the polystyrene materials with hierarchically porous interconnected pores. ${ }^{34}$

Herein, we report a novel and facile processing technique to fabricate polystyrene solid foam, derived from styrene-based HIPEs emulsified by Span 20 together with $\mathrm{Fe}_{3} \mathrm{O}_{4}$. The micromorphology of the polystyrene foam was characterized to show the hierarchically interconnected pore structures. Thermal stability, magnetic properties, hydrophobicity, as well as oleophilicity of the composite materials were evaluated. Importantly, the oil adsorption capacity and hydrophobicity of the polystyrene composite remained high even after 10 cycles of adsorption and regeneration. The selectivity of the material towards oil and water reached around 99\%. Moreover, the material was regenerated by centrifugation and approximately $90 \%$ oil could be recovered. As a result, the material was confirmed to be an efficient oil adsorbent.

\section{Experimental methods}

\subsection{Chemicals and materials}

Span 20 (with HLB value 8.6) and stain Sultan IV were purchased from Sinopharm Chemical Reagent Co. Ltd., Shanghai. St and azobisisobutyronitrile (AIBN) were provided by Lingfeng Chemical Reagent Co. Ltd., Shanghai. DVB and cubic crystal $\mathrm{Fe}_{3} \mathrm{O}_{4}$ were purchased from Aladdin. Absolute ethanol was purchased from Nanjing Chemical Reagent Co. Ltd., Nanjing. Deionized water was used in all the experiments. All the chemicals were of reagent grade and used in this study without any further purification.

\subsection{Preparation of HIPEs and synthesis of the polystyrene foam}

After a great number of trials, three representative experiments were carried out to form the porous solid foams, and the experimental details are presented herein for further investigation (Table 1). For preparing the HIPEs, certain amounts of dried $\mathrm{Fe}_{3} \mathrm{O}_{4}$ particles and Span 20, as well as $0.01 \mathrm{~g}$ of oil-soluble initiator of AIBN, were dispersed in the oil phase consisting of
Table 1 Composition of the HIPEs

\begin{tabular}{llllll}
\hline Sample & Span 20/g & $\mathrm{Fe}_{3} \mathrm{O}_{4} / \mathrm{g}$ & $\mathrm{St} / \mathrm{g}$ & $\mathrm{DVB} / \mathrm{g}$ & Water/mL \\
\hline 1 & 0.050 & 0.06 & 0.4 & 0.1 & 6 \\
2 & 0.050 & 0.06 & 0.4 & 0.1 & 8 \\
3 & 0.075 & 0.08 & 0.4 & 0.1 & 8 \\
\hline
\end{tabular}

$0.4 \mathrm{~g}$ of St and $0.1 \mathrm{~g}$ of DVB via ultrasonication for $10 \mathrm{~min}$. After this, deionized water was added stepwise into the abovementioned mixture. Vigorous shaking by hand was performed for the preparation of viscous and homogeneous emulsions after the addition of each batch of water until the final HIPEs were formed, which generally took about $5 \mathrm{~min}$. Then, the HIPEs were put in an oven, and the organic monomers of St and DVB polymerized at $70{ }^{\circ} \mathrm{C}$ in $8 \mathrm{~h}$. After polymerization, the residual Span 20 and organic monomers in the PolyHIPEs were removed by Soxhlet extraction with ethanol. Finally, the resulting monolithic solid foam was dried at $70{ }^{\circ} \mathrm{C}$ for $4 \mathrm{~h}$ using an oven until constant weights were achieved.

\subsection{Characterization}

A Model-S4800 field-emission scanning electron microscope (SEM, Hitachi) operating at $15 \mathrm{kV}$ was used to investigate the microstructures of the PolyHIPEs. X-ray diffraction (XRD) analysis was performed using a diffractometer (Bruker D8 Super Speed) with $\mathrm{Cu} \mathrm{K}_{\alpha}$ radiation. Pore size distribution of the polymer foam was obtained via mercury porosimetry (Autopore IV 9510, Micromeritics, USA). The energy-dispersive X-ray spectroscopy (EDS) measurements were carried out using an Oxford Inca Energy spectrometer. The thermogravimetric (TG) analyzer TA 2100 provided by TA instruments (USA) was used for thermogravimetric analysis. All the samples were heated from 50 to $600{ }^{\circ} \mathrm{C}$ at the heating rate of $10{ }^{\circ} \mathrm{C} \min ^{-1}$. Moreover, the magnetic properties of the PolyHIPEs were obtained using a vibrating sample magnetometer (VSM) Lakeshore 735 at $300 \mathrm{~K}$ and a field of 8000 Oe. Water contact angles (WCAs) at room temperature were obtained by a contact angle instrument (XGCAMB). A $4 \mu \mathrm{L}$ aliquot of deionized water was placed on the surface of the sample each time and then the WCAs were obtained. The monolithic material was regenerated by a centrifuge (TGL-15B) to remove the adsorbed oil from the PolyHIPEs. An Agilent Technologies Cary $100 \mathrm{UV}$-Vis spectrometer was used to detect the concentration of the residual oil in the oil/water mixture.

\subsection{Oil-water separation}

Oil adsorption capacities of the polystyrene material towards diesel, edible oil, and lubricating oil were measured. About $0.15 \mathrm{~g}$ of sample was put into the oil. After adsorption saturation, the monolithic polystyrene composite material soaked with oil was removed, and the weight was obtained. Then, the value $k$ was calculated according to the following equation:

$$
k=\left(M_{\mathrm{sat}}-M_{0}\right) / M_{0}
$$


where $k$ represents the oil adsorption capacity, $M_{\text {sat }}$ is the weight of the foam after saturation adsorption of oil, and $M_{0}$ is the mass of the original dried foam. For each type of foam, 3 measurements were conducted, and then, the average value was calculated as the final oil adsorption capacity. After each adsorption experiment, centrifugation was used to remove the oil from the foam, and then, the material was ready to be used for the next cycle of adsorption.

The selectivity $S$ of the materials for oil-water mixtures was calculated according to the following equation:

$$
S(\%)=\left(M_{\mathrm{i}}-M_{\mathrm{r}}\right) /\left(M_{\mathrm{sat}}-M_{0}\right)
$$

where $M_{\mathrm{i}}$ represents the initial oil weight of the oil/water mixture and $M_{\mathrm{r}}$ is the residual oil in the oil/water mixture after adsorption of the material. Residual oil was obtained by extraction and then analyzed by UV-vis spectrometry. ${ }^{34}$

\section{Results and discussion}

\section{1. $\mathrm{Fe}_{3} \mathrm{O}_{4}$ particles and emulsion}

The raw material $\mathrm{Fe}_{3} \mathrm{O}_{4}$ particles were cubic crystals with the size of about $200 \mathrm{~nm}$, as shown in Fig. 1(a). IR characterization and the water contact angle of the $\mathrm{Fe}_{3} \mathrm{O}_{4}$ particles are shown in Fig. $\mathrm{S} 1$ and $\mathrm{S} 2, \uparrow$ respectively. Moreover, via control experiments, it was found that neither Span 20 nor $\mathrm{Fe}_{3} \mathrm{O}_{4}$ particles alone were suitable to stabilize the water-in-oil emulsions. As a result, the introduction of $\mathrm{Fe}_{3} \mathrm{O}_{4}$ particles served two purposes: it stabilized the emulsion and imparted the resulting polymer material with magnetic properties. The obtained system was gel-like emulsion, as shown in Fig. 1(b).

\subsection{Morphology and pore size distribution of the polystyrene foam}

During the formation of HIPEs, water was dispersed in the emulsions with the assistance of Span 20 together with the solid particles of $\mathrm{Fe}_{3} \mathrm{O}_{4}$, resulting in a stable water-in-oil system. Then, the organic continuous phase St and DVB polymerized, causing solidification of the microstructures of the HIPEs. Finally, hierarchically porous PolyHIPEs foams were obtained after the removal of the internal water phase. Because PolyHIPE is a replica of the HIPE structure at the gel point of polymerization, ${ }^{35}$ water droplets in the emulsions before polymerization led

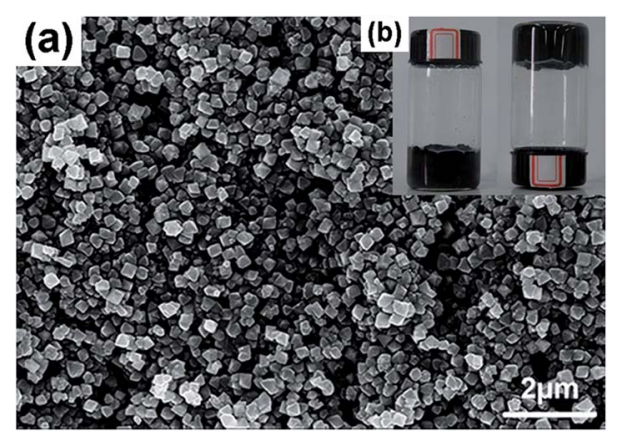

Fig. 1 (a) $\mathrm{Fe}_{3} \mathrm{O}_{4}$ particles and (b) obtained emulsions. to the formation of voids after solidification. ${ }^{36}$ Moreover, it is believed that the thin oil film retracted at the polymerization gel time, which led to the formation of holes or windows between adjacent voids. ${ }^{24,37}$ In addition, it is a common phenomenon in polymer chemistry that shrinkage during polymerization causes the formation of the interconnected holes.

As shown in Fig. 2(a) and (b), increase in the water content was found to cause an obvious increase in the average void diameter of the resulting polymer materials. However, the density of the material significantly decreased from $0.081 \mathrm{~g}$ $\mathrm{cm}^{-3}$ to $0.063 \mathrm{~g} \mathrm{~cm}^{-3}$. This phenomenon was correlated with the original compositions of the emulsions from which they were fabricated. Voids in the PolyHIPE were derived from the water droplets in the emulsions before polymerization. ${ }^{36}$ As a result, a higher water content led to the formation of larger voids of the PolyHIPE. It has also been suggested that the internal structures and densities of the resulting composite material can be adjusted by the water content. ${ }^{38}$ By optimizing the experimental conditions, sample 3 was finally prepared. As shown in Fig. 2(c), the obtained polymer material displayed a homogeneous morphology with more developed interconnected pores, which was beneficial for the transportation of the oily liquid inside the material. Furthermore, a hierarchically porous structure with small pores from several hundred nanometers to several micrometers in the void walls was constructed. It showed that sample 3 had a relatively small void size, which was due to the increased concentration of Span 20 and $\mathrm{Fe}_{3} \mathrm{O}_{4}$ particles. Both of them can strongly adsorb at the oil-water interface, preventing the droplets from coalescence and improving the stability of the water-in-oil emulsion. ${ }^{39-42}$

The enlarged view also showed that the voids and pores of the PolyHIPEs were decorated with $\mathrm{Fe}_{3} \mathrm{O}_{4}$ particles and thus were functionalized with magnetic properties, which were identified by further characterizations. The raw material $\mathrm{Fe}_{3} \mathrm{O}_{4}$ particles were cubic. However, they tended to be spherical in the obtained PolyHIPEs. A possible explanation was that the organic monomers St and DVB adsorbed at the surface of the $\mathrm{Fe}_{3} \mathrm{O}_{4}$ particles and then polymerized, making the particles spherical.

The density of sample 3 was $0.065 \mathrm{~g} \mathrm{~cm}^{-3}$ and the porosity was $91.7 \%$. Moreover, the pore size distribution of sample 3 was obtained by mercury intrusion instruments, and three distinct peaks were found, as shown in Fig 3 . The peaks at around $10 \mu \mathrm{m}$ and $20 \mu \mathrm{m}$ can be attributed to the voids, whereas the peak at around $2 \mu \mathrm{m}$ was ascribed to the pores on the wall of the voids. Moreover, the pore size distribution result correlated well with the SEM image. Note that the hierarchical pore structure with nano- and micron-size was beneficial for the improvement of the hydrophobicity of the material.

\subsection{Elemental analysis of the polystyrene foam}

The XRD diffraction patterns of pure $\mathrm{Fe}_{3} \mathrm{O}_{4}$ particles, sample 1, sample 2, and sample 3 are shown in Fig. 4(a). The representative diffraction peaks of the $\mathrm{Fe}_{3} \mathrm{O}_{4}$ crystal can be characterized by (220), (311), (400), (511), and (440) planes (JCPDS no. 19-0629). Moreover, these representative peaks can still be observed in the 

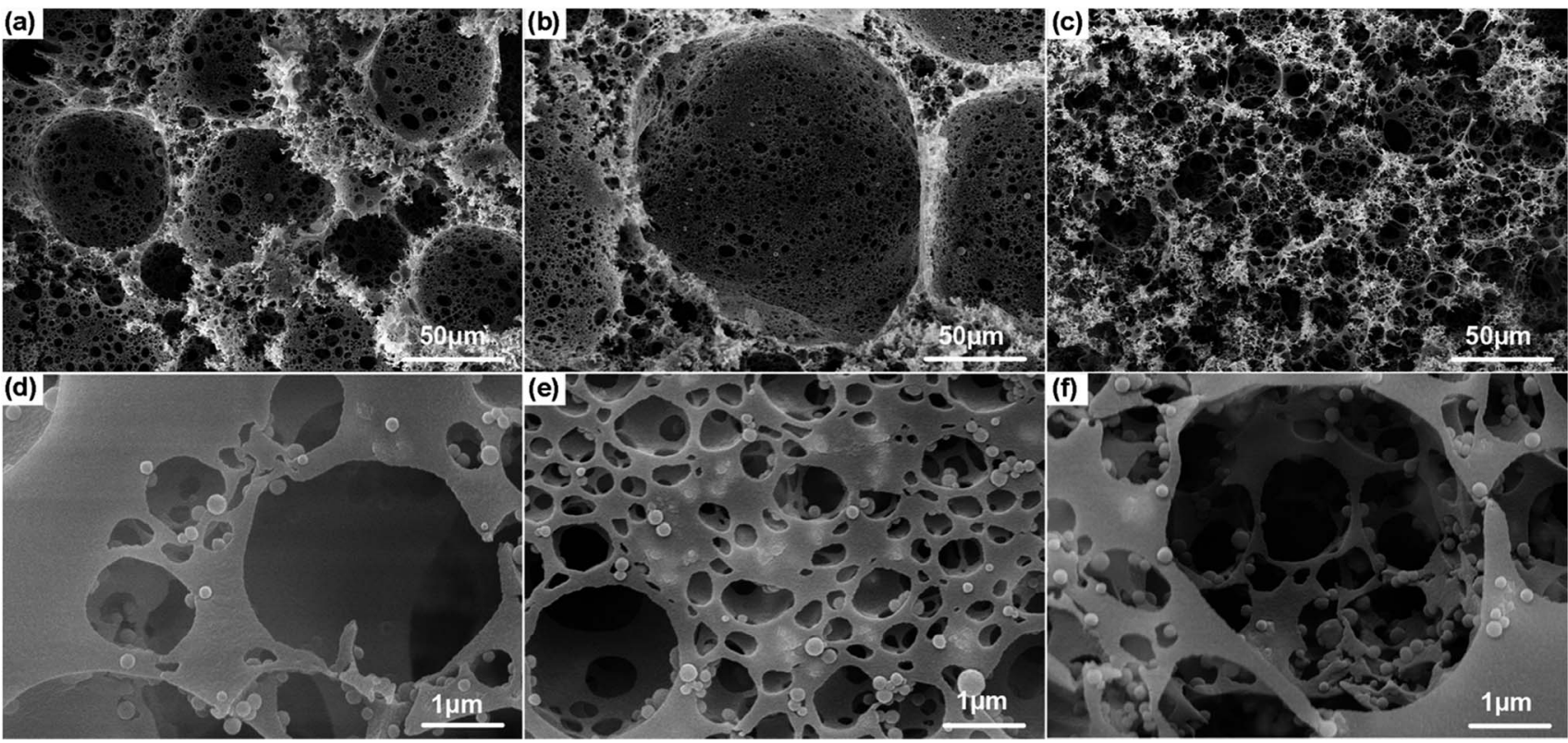

Fig. 2 SEM images of (a) sample 1, (b) sample 2, and (c) sample 3; (d), (e), and (f) are the enlarged view of (a), (b), and (c), respectively.

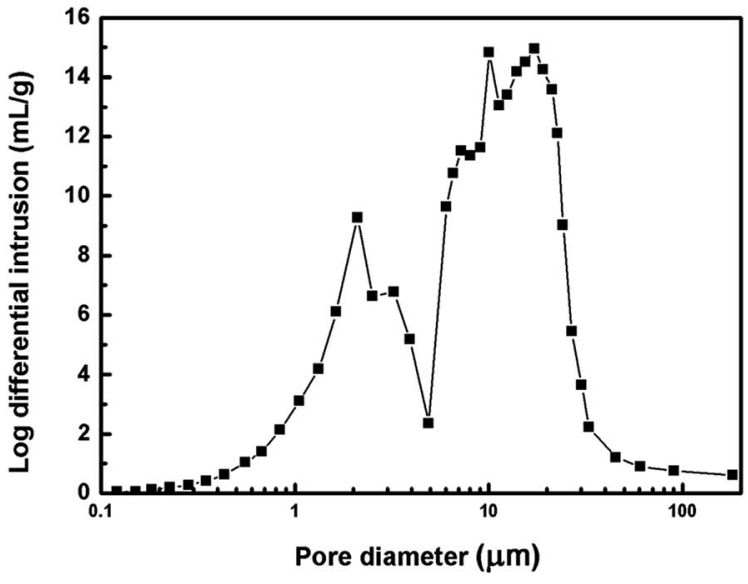

Fig. 3 Pore size distribution of sample 3.

PolyHIPE material. In addition, except for the diffraction peaks at $30.1^{\circ}, 35.7^{\circ}, 43.1^{\circ}, 57.2^{\circ}$, and $62.6^{\circ}$ for $\mathrm{Fe}_{3} \mathrm{O}_{4}{ }^{43}$ no other peak appeared in the composite material. This could ensure that the crystalline structure of $\mathrm{Fe}_{3} \mathrm{O}_{4}$ did not change, and no impurity was introduced during the preparation process. As shown in Fig. 4(b), EDS was carried out to confirm the existence of C, Fe, and $\mathrm{O}$ elements in the polymer foam. Elemental mapping (Fig. 4(c)) showed the homogeneous distribution of the three types of elements in the polystyrene material. XRD, EDS, and elemental mapping results were consistent, and all these characterizations together indicated that the magnetic $\mathrm{Fe}_{3} \mathrm{O}_{4}$ particles were dispersed in the resulting polymer backbone.

\subsection{Thermal stability of the polystyrene foam}

To measure the $\mathrm{Fe}_{3} \mathrm{O}_{4}$ content in the PolyHIPE material, TG analysis was performed. As shown in Fig. 5, sample 1 (curve a) and sample 2 (curve b) showed a weight loss of $81.6 \%$ and $80.2 \%$ in the temperature range of $50-600{ }^{\circ} \mathrm{C}$, which was attributed to the decomposition of polystyrene. For the $\mathrm{Fe}_{3} \mathrm{O}_{4}$ residue at $600{ }^{\circ} \mathrm{C}$, sample 3 (curve c) maintained $24.5 \mathrm{wt} \%$, obviously higher than that of sample 1 and sample 2, due to the addition of more $\mathrm{Fe}_{3} \mathrm{O}_{4}$ before the polymerization of the HIPEs. Moreover, all three samples began to decompose when the temperature was over $220{ }^{\circ} \mathrm{C}$. Weight loss of sample 3 appeared even after $300{ }^{\circ} \mathrm{C}$. It seemed that the higher $\mathrm{Fe}_{3} \mathrm{O}_{4}$ content contributed to a higher decomposition temperature. The abovementioned test results verified that the polystyrene material had an excellent thermal stability. There was no doubt that this good stability ensured that the composite material could be used at high temperature during the oil/water separation.

\subsection{Magnetic property of the polystyrene foam}

Fig. 6 shows the magnetic properties of sample 1, sample 2, and sample 3 at room temperature, with the saturation magnetism of $15.4,17.8$, and $22.5 \mathrm{emu}^{-1}$, respectively. The raw material $\mathrm{Fe}_{3} \mathrm{O}_{4}$ particles exhibited a strong magnetic property and the saturation magnetism was $87.9 \mathrm{emu}^{-1}$, as shown by curve d. The magnetism of the composite polystyrene foam showed a decrease compared to that of the pure $\mathrm{Fe}_{3} \mathrm{O}_{4}$ particles, due to the non-magnetic polystyrene material. The abovementioned results also indicated that the $\mathrm{Fe}_{3} \mathrm{O}_{4}$ particles were dispersed in the network of the resulting PolyHIPE material, and the composite materials were successfully functionalized by the magnetic particles as well. Furthermore, the VSM result was consistent with the concentrations of the $\mathrm{Fe}_{3} \mathrm{O}_{4}$ particles in the samples, as characterized by the TG analysis. The introduction of $\mathrm{Fe}_{3} \mathrm{O}_{4}$ particles endowed the PolyHIPE composite material with additional magnetic properties, which ensured that the polymer foam could be manipulated via an external magnetic field during applications. 


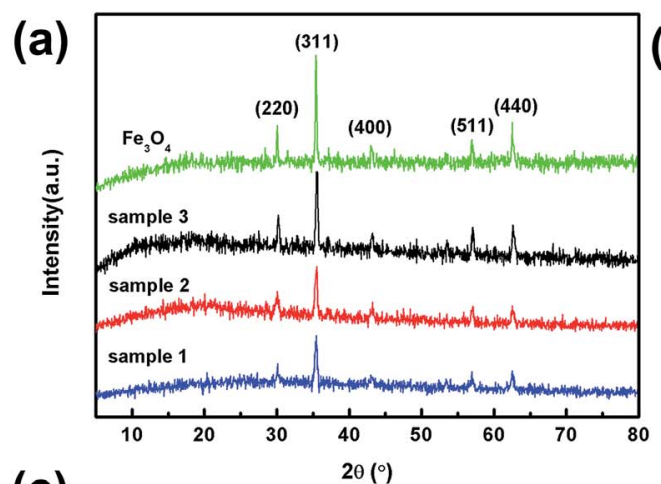

(b)

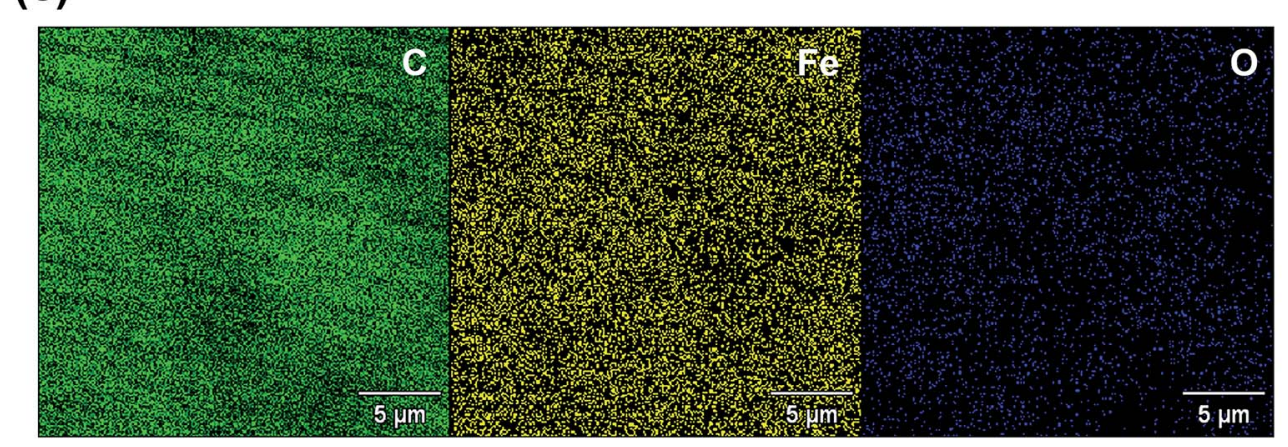

Fig. 4 (a) XRD analysis of pure $\mathrm{Fe}_{3} \mathrm{O}_{4}$ particles, sample 1, sample 2, and sample 3; (b) EDS spectra of sample 3; (c) elemental mapping of sample 3.

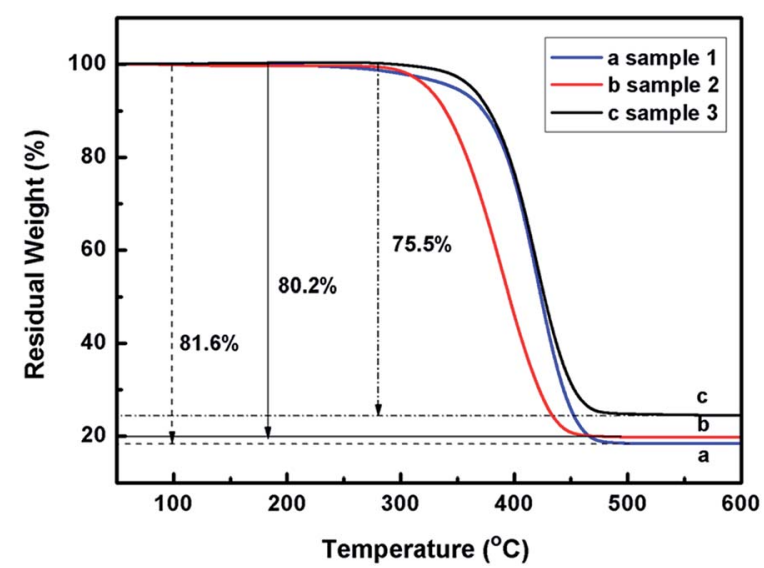

Fig. 5 TG analysis of (a) sample 1, (b) sample 2, and (c) sample 3.

\subsection{Hydrophobicity and oleophilicity of the polystyrene foam}

The polystyrene foam had excellent hydrophobicity with WCA approaching $147^{\circ}$, as shown in Fig. 7 (a). Fig. 7 (b) shows that the monolithic foam can successfully float on water surface, due to the low density and water repellence of the material. The contact angle between diesel and the composite material was nearly $0^{\circ}$ (Fig $7(\mathrm{c})$ ). When a drop of diesel was placed on the monolithic surface, it was immediately adsorbed and penetrated into the material, indicating the superior lipophilicity of the obtained material. In addition, hydrophobicity and oleophilicity provided selectivity to the material. When used in oil/ water separation, it can adsorb oil and repel water. Moreover, the selectivity of the material was as high as $99.1 \%, 98.7 \%$, and

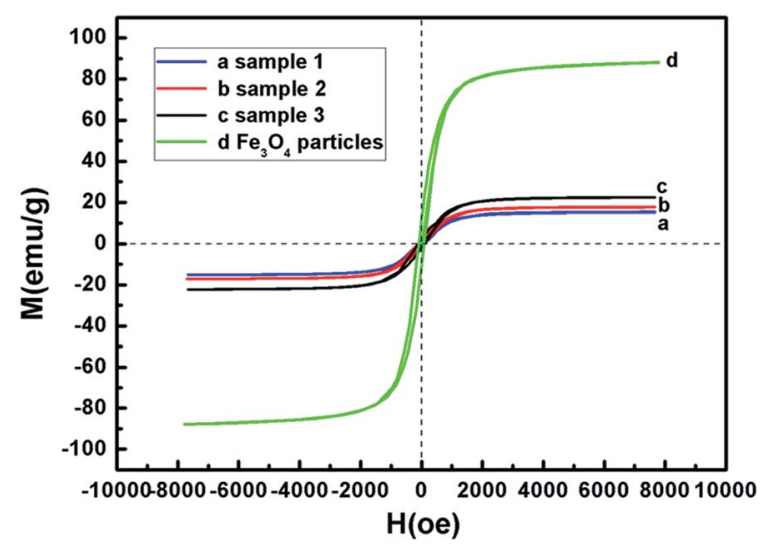

Fig. 6 VSM curves of (a) sample 1, (b) sample 2, (c) sample 3, and (d) pure $\mathrm{Fe}_{3} \mathrm{O}_{4}$ particles.

99.3\% towards diesel, edible oil, and lubricating oil respectively. Considering practical applications, it is very important for an adsorption material to possess the abovementioned property.

\subsection{Oil adsorption process and regeneration of the polystyrene foam}

Fig. 8(a) is a simulation of the oil removal process by the polystyrene material. Approximately $1.5 \mathrm{~g}$ of diesel was dropped into water and it quickly spread on the water surface. Then, $0.163 \mathrm{~g}$ of polystyrene foam was added into the mixture of oil and water. The foam material can be driven to the oil zone under the control of a magnet, and the diesel immediately penetrated into the foam. After adsorption, the water surface became clear again, and the low density ensured that the foam soaked with 
(a)

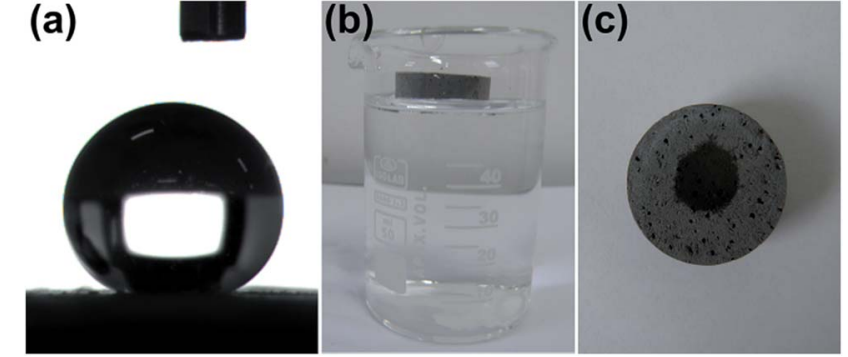

Fig. 7 (a) Water contact angle of the polystyrene foam; (b) the polystyrene foam floating on the water surface; and (c) a drop of diesel adsorbed into the polystyrene material.
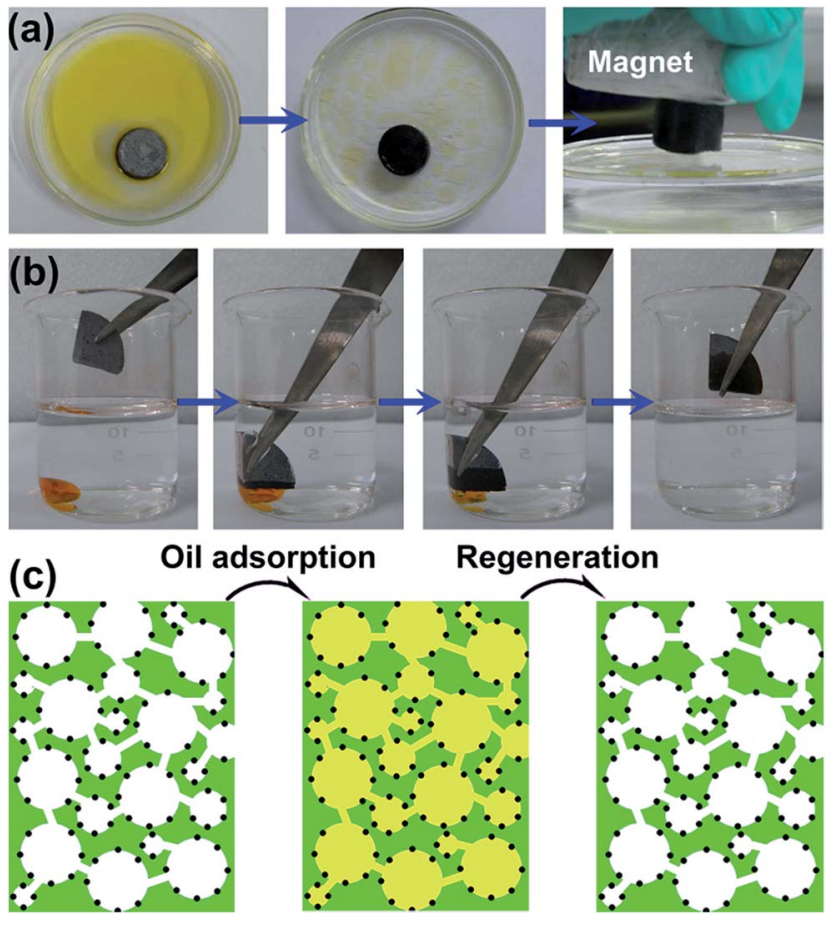

Regeneration

Fig. 8 (a) Removal of diesel from the water surface by the polystyrene foam under an external magnetic field; (b) images of the removal process of tetrachloromethane (dyed with stain Sultan IV for clear observation) sinking below the water surface; and (c) schematic of the oil adsorption and regeneration process of the polystyrene foam.

diesel could still float on the water surface. Then, the foam was conveniently obtained and removed from water by a magnet, avoiding secondary pollution caused by the residual oil adsorption material in water. Polystyrene foam could repel water and adsorb the organic liquid, having high density, that was sinking below the water surface, as shown in Fig. 8(b). When the monolithic foam was completely immersed in water by the external force, a transparent air film, formed by a continuous air layer between the highly hydrophobic surface of the material and water, on the material surface was observed due to the non-wetting behavior. ${ }^{44}$ Fig. 8 (c) shows the process of oil adsorption and the regeneration of the porous polystyrene material. When oil first contacted the composite material, the surface of the solid foam was wetted by the oil due to van der
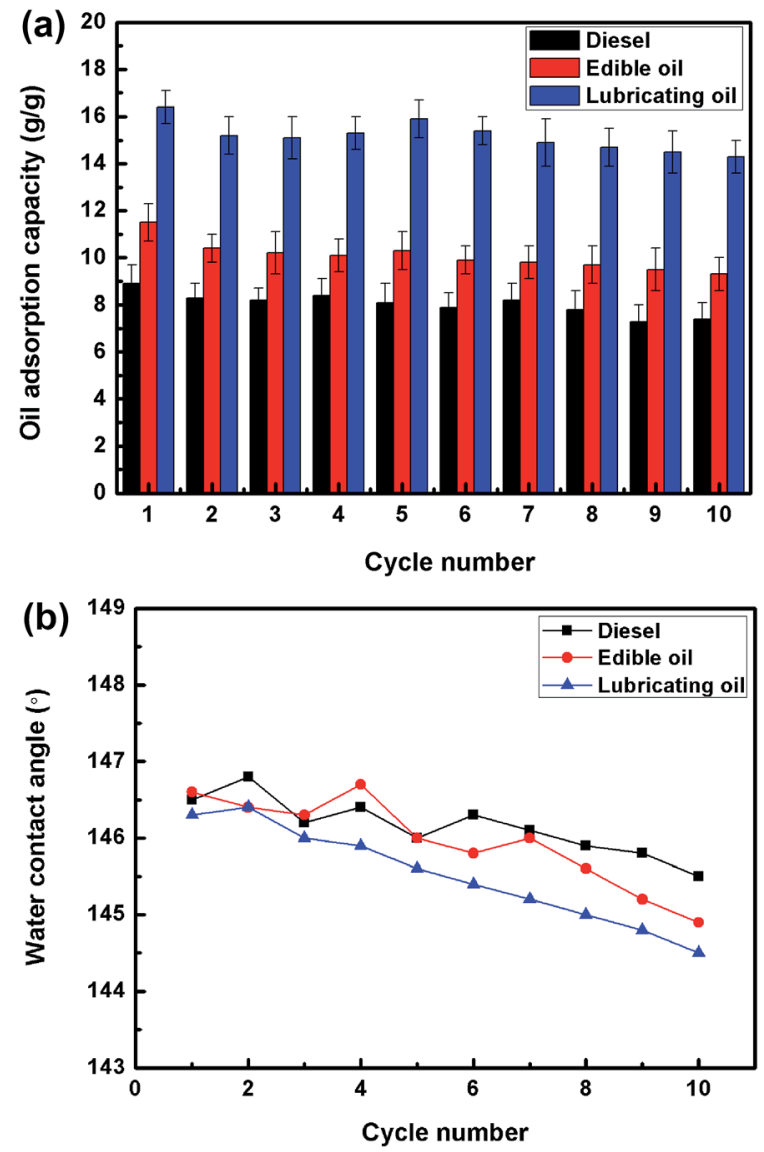

Fig. 9 (a) Oil adsorption capacity of sample 3 for cyclic oil-water separation and (b) water contact angle of sample 3 after every oil/ water separation cycle.

Waals forces between the polystyrene foam and the oil. ${ }^{45}$ Then, the oil was further driven into the inner open pores by capillary action. ${ }^{46}$ Therefore, it is not difficult to understand that the interconnected pore structures were beneficial for the improvement of the oil adsorption rate as well as the oil adsorption capacity. After adsorption saturation, centrifugation was adopted to counteract the force between the material and oil and to empty the oil in the inner pores. Finally, the polymer foam was regenerated and was ready for the next cycle of oil adsorption.

\subsection{Oil adsorption capacity and the reusability of the polystyrene foam}

As shown in Fig. 9(a), the adsorption capacity of polystyrene foam towards diesel, edible oil, and lubricating oil was investigated. Oil adsorption capacities of sample 1, sample 2, and sample 3 towards lubricating oil were $7.9 \mathrm{~g} \mathrm{~g}^{-1}, 8.3 \mathrm{~g} \mathrm{~g}^{-1}$ and $16.4 \mathrm{~g} \mathrm{~g}^{-1}$, respectively. As a result, the oil adsorption capacity and reusability of sample 3 was further investigated. The weight gains of sample 3 were $8.9,11.5$, and 16.4 times its original mass for the three different types of oils. It is clear that the oil intake capacity of the material was dependent on the density and viscosity of the target oil pollutants. After adsorption saturation, 
the polystyrene foam soaked with oil was regenerated by centrifugation and was then directly used for the next cycle of oil adsorption. The oil removal rate is shown in Fig. S3. $\dagger$ After 10 cycles, the adsorption capacity was still at a high level with a slight decrease. Fig. 9(b) shows that water contact angle decreased with an increase in the cycle number. However, the hydrophobicity was retained after 10 cycles, and the contact angle remained over $144.5^{\circ}$. As a result, the composite material showed excellent reusability; this makes it a competitive candidate for the separation of oil from water in practical applications.

\section{Conclusions}

Here, we report an easily controlled and flexible method to prepare a hierarchically porous monolithic polystyrene material via polymerization of an organic phase and removal of the internal water phase. Span 20 and $\mathrm{Fe}_{3} \mathrm{O}_{4}$ particles were held at the interface to act as a barrier to prevent Ostwald ripening, which proved to be an effective co-stabilizer of the St-DVB HIPEs template. Moreover, this two-component stabilizer system also provided a new possibility to influence the microstructures of the resulting PolyHIPEs material. Pronounced variations in microstructure could be achieved just by adjusting the concentration of water, Span 20 or $\mathrm{Fe}_{3} \mathrm{O}_{4}$ particles. In principle, the method reported herein may be conveniently extended to fabricate other porous composite PolyHIPE materials adopting various inorganic nanoparticles. The composite material showed superior reusability, and the oil adsorption capacity only slightly decreased even after 10 cycles. Furthermore, the composite PolyHIPE material also exhibited excellent thermal stability, magnetic properties, oleophilicity, as well as hydrophobicity. In conclusion, all these features together make it an ideal candidate in real oil/water separation applications.

\section{Acknowledgements}

This work was financially supported by the Natural Science Foundation of China (50972060), Weapon Research Support Fund (62201070804), Qing Lan Project, Environmental Protection Scientific Research Project of Jiangsu Province (2016056), Project funded by the Priority Academic Program Development of Jiangsu Higher Education Institutions, Shanghai Aerospace Science and Technology Innovation Fund (SAST2015020), and Basic Product Innovation Technology Research Project of Explosives.

\section{Notes and references}

1 Y. L. Shi, Y. Wu, X. J. Feng, Y. S. Wang, G. R. Yue and S. P. Jin, Appl. Surf. Sci., 2016, 367, 493-499.

2 C. An, G. Huang, Y. Yao and S. Zhao, Sci. Total Environ., 2017, 579, 537-556.

3 O. S. H. Santos, M. Coelho da Silva, V. R. Silva, W. N. Mussel and M. I. Yoshida, J. Hazard. Mater., 2017, 324, 406-413.

4 B. Wang, W. Liang, Z. Guo and W. Liu, Chem. Soc. Rev., 2015, 44, 336-361.
5 R. Wu, B. Yu, X. Liu, H. Li, W. Wang, L. Chen, Y. Bai, Z. Ming and S. T. Yang, Appl. Surf. Sci., 2016, 362, 56-62.

$6 \mathrm{~J}$. Hu, W. Yuan, W. Chen, X. Xu and Y. Tang, Appl. Surf. Sci., 2016, 389, 1192-1201.

7 J. Q. Hu, S. Z. Yang, L. Guo, X. Xu, T. Yao and F. Xie, J. Mol. Liq., 2017, 227, 351-355.

8 P. Madhusudhana Reddy, C. J. Chang, J. K. Chen, M. T. Wu and C. F. Wang, Appl. Surf. Sci., 2016, 368, 27-35.

9 J. Pan, J. Zeng, Q. Cao, H. Gao, Y. Gen, Y. Peng, X. Dai and Y. Yan, Chem. Eng. J., 2016, 284, 1361-1372.

10 Z. Li, J. Zhang, T. Luo, X. Tan, C. Liu, X. Sang, X. Ma, B. Han and G. Yang, Soft Matter, 2016, 12, 8841-8846.

11 T. Zhang and Q. Guo, Chem. Commun., 2016, 52, 4561-4564.

12 Y. Zhu, W. Wang, Y. Zheng, F. Wang and A. Wang, Carbohydr. Polym., 2016, 140, 51-58.

13 A. Carranza, K. Song, J. F. A. Soltero-Martinez, Q. Wu, J. A. Pojman and J. D. Mota-Morales, $R S C A d v$., 2016, 6, 81694-81702.

14 Y. Luo, A.-N. Wang and X. Gao, Soft Matter, 2012, 8, 7547. 15 Y. Zhu, Y. Zheng, L. Zong, F. Wang and A. Wang, Carbohydr. Polym., 2016, 149, 242-250.

16 A. Khodabandeh, R. Dario Arrua, C. T. Desire, T. Rodemann, S. A. F. Bon, S. C. Thickett and E. F. Hilder, Polym. Chem., 2016, 7, 1803-1812.

17 P. Pakeyangkoon, R. Magaraphan, P. Malakul and M. Nithitanakul, Macromol. Symp., 2008, 264, 149-156.

18 N. Zhang, S. Zhong, X. Zhou, W. Jiang, T. Wang and J. Fu, Chem. Eng. J., 2016, 298, 117-124.

19 A. Barbetta and N. R. Cameron, Macromolecules, 2004, 37, 3202-3213.

20 V. O. Ikem, A. Menner and A. Bismarck, Angew. Chem., Int. Ed., 2008, 47, 8277-8279.

21 T. Zhang and Q. Guo, Chem. Eng. J., 2017, 307, 812-819.

22 Y. Zhang, Y. Shen, Y. Chen, Y. Yan, J. Pan, Q. Xiong, W. Shi and L. Yu, Chem. Eng. J., 2016, 294, 222-235.

23 Y. Qian, T. Wang, X. Qiu, D. Zhao, D. Liu and Y. Deng, ACS Sustainable Chem. Eng., 2016, 4, 7193-7199.

24 A. Carranza, M. G. Pérez-García, K. Song, G. M. Jeha, Z. Diao, R. Jin, N. Bogdanchikova, A. F. Soltero, M. Terrones, Q. Wu, J. A. Pojman and J. D. Mota-Morales, ACS Appl. Mater. Interfaces, 2016, 8, 31295-31303.

25 M. J. Rymaruk, K. L. Thompson, M. J. Derry, N. J. Warren, L. P. D. Ratcliffe, C. N. Williams, S. L. Brown and S. P. Armes, Nanoscale, 2016, 8, 14497-14506.

26 X. Song, G. Yin, Y. Zhao, H. Wang and Q. Du, J. Polym. Sci., Part A: Polym. Chem., 2009, 47, 5728-5736.

27 Y. Zhao, H. Wang, X. Song and Q. Du, Macromol. Chem. Phys., 2010, 211, 2517-2529.

28 V. O. Ikem, A. Menner, T. S. Horozov and A. Bismarck, Adv. Mater., 2010, 22, 3588-3592.

29 A. Vílchez, C. Rodriguez-Abreu, A. Menner, A. Bismarck and J. Esquena, Langmuir, 2014, 30, 5064-5074.

30 S. Kovacic, N. B. Matsko, G. Ferk and C. Slugovc, J. Mater. Chem. A, 2013, 1, 7971-7978.

31 W. Yi, H. Wu, H. Wang and Q. Du, Langmuir, 2016, 32, 982990. 
32 Z. Hu, S. Ballinger, R. Pelton and E. D. Cranston, J. Colloid Interface Sci., 2015, 439, 139-148.

33 B. P. Binks and S. O. Lumsdon, Langmuir, 2000, 16, 25392547.

34 S. Yu, H. Tan, J. Wang, X. Liu and K. Zhou, ACS Appl. Mater. Interfaces, 2015, 7, 6745-6753.

35 K. J. Lissant, B. W. Peace, S. H. Wu and K. G. Mayhan, J. Colloid Interface Sci., 1974, 47, 416-423.

36 Y. Luo, A. N. Wang and X. Gao, Soft Matter, 2012, 8, 18241830.

37 N. R. Cameron, D. C. Sherrington, L. Albiston and D. P. Gregory, Colloid Polym. Sci., 1996, 274, 592-595.

38 P. Jing, X. Fang, J. Yan, J. Guo and Y. Fang, J. Mater. Chem. A, 2013, 1, 10135-10141.

39 R. X. Bai, L. H. Xue, R. K. Dou, S. X. Meng, C. Y. Xie, Q. Zhang, T. Guo and T. Meng, Langmuir, 2016, 32, 92549264.
40 J. Jiang, Y. Ma, Z. Cui and B. P. Binks, Langmuir, 2016, 32, 8668-8675.

41 L. Peng, A. Feng, S. Liu, M. Huo, T. Fang, K. Wang, Y. Wei, X. Wang and J. Yuan, ACS Appl. Mater. Interfaces, 2016, 8, 29203-29207.

42 A. Marefati, M. Bertrand, M. Sjöö, P. Dejmek and M. Rayner, Food Hydrocolloids, 2017, 63, 309-320.

43 L. Yu, X. Zhou and W. Jiang, Ind. Eng. Chem. Res., 2016, 55, 9498-9506.

44 I. A. Larmour, S. E. J. Bell and G. C. Saunders, Angew. Chem., Int. Ed., 2007, 46, 1710-1712.

45 N. Zhang, W. Jiang, T. Wang, J. Gu, S. Zhong, S. Zhou, T. Xie and J. Fu, Ind. Eng. Chem. Res., 2015, 54, 11033-11039.

46 N. Chen and Q. Pan, ACS Nano, 2013, 7, 6875-6883. 